\title{
On the representation of certain digit sequences in memory
}

\author{
ROBERT E. WARREN and MICHAEL HESS \\ Columbia University, New York, New York 10027
}

\begin{abstract}
The representation in memory of digit strings such as dates was examined by observing the delays produced in color naming in a discrete-trial Stroop task when these sequences were used as carriers for the color. In Experiment I, subjects were presented on each trial with either a four-digit sequence in color, or a patch of color, and required to name the color as quickly as possible. Digit sequence trials produced significantly longer color-naming latencies. In Experiment II, five different types of four-digit sequences were used: dates, odd or even series, ascending sequences, descending sequences and repetitions. Trials using a double row of dashes in color served as the control. Only two types of digit series produced color-naming latencies significantly longer than those observed for dashes: dates, e.g., 1776, and odd or even series, e.g., 2468. It was argued that the significant delays over dash-trial color-naming latencies produced by these digit sequences indicated their functioning as cohesive units in memory, much like those hypothesized for letter sequences that form words.
\end{abstract}

Recent descriptions of the representation of numbers in memory have emphasized the structural aspects of their storage and retrieval. Indeed, Posner and Warren (1972) have suggested that ordered sequences such as the alphabet and the number system represent veridical models of the simplest type of structure used to store material in memory. Several studies have produced evidence for the operation of the memorial representation of the number system as an ordered list. Buschke and Hinrichs (1968), for example, have shown that errors in the missing scan procedure are not randomly distributed but follow from the ordered nature of the stored digit series. In the missing scan task, subjects are given in random order all but one of a linear sequence of numbers and are required to produce the missing digit. Errors of commission in this test tend to fall close to the target in terms of the linear sequence of numbers used. If the sequence of Numbers 13-25 is used and 19 is the target, for example, 18 and 20 are more common errors than 17 and 21.

Similarly, Marcel and Forrin (1974) have shown that in an eight-choice reaction time task, using the digits 2 through 9 as stimuli, reaction time to a digit varies as a function of the identity of the digit seen on the immediately prior trial. The closer the prior digit is to the target on the current trial in terms of the linear sequence of numbers, the faster the subject's reaction time. If 6 is preceded by 5 , for example, reaction time is faster than if it is preceded by 4 .

While the operation of the number system as a single ordered series in memory is apparent from these studies, it must be the case that some specific groups of numbers are represented as uniquely ordered units in memory as well. Memory for such digit groups as dates, telephone

Requests for reprints should be sent to $R$. E. Warren, Department of Psy chology, Columbia University, New York, New York 10027. numbers, and license plate designations would presumably be of this latter sort.

Just as sequences of letters appear to be stored as single units in memory when they form a word, so, it could be argued, must sequences of digits be stored as single units when they form a meaningful group such as a date. If this is the case, then sequences of this type should share some of the characteristics of words when introduced into certain tasks.

In the case of letter sequences, it has been shown that the degree to which the component letters form a recognizable word in English is directly related to the amount of interference the sequence produces when used as a base item in the Stroop color-word task (Klein, 1964). In the Stroop task (Stroop, 1938), words are presented printed in colored inks, and the subject is asked to name the color of ink as fast as possible. Color-naming latencies are shortest when the carrier for the color is a uniform patch or series of dashes. Meaningless sequences of jumbled letters produce slightly longer latencies, and, when words serve as the carriers, color-naming times increase as the frequency of the base item in English increases (Klein, 1964).

If digit sequences such as dates are represented in memory as cohesive units of the same sort as words, it should follow that they, too, should produce interference in the Stroop task when used as carriers for the color. Two experiments were conducted to test this hypothesis. In Experiment I, the color-naming latencies for trials using digit sequences as carriers were compared with those using a uniform patch of color.

\section{EXPERIMENT I}

\section{Method}

Subjects. Twenty-seven Columbia University undergraduates served individually as subjects in a single $1 / 2-\mathrm{h}$ session. They received supplementary course credit for their participation. 
Table 1

Color-Naming Latencies of the Various Stimulus Types

\begin{tabular}{lc}
\hline Stimulus Type & Mean Latency \\
\hline Dates & 701 \\
Odd-Even & 698 \\
Descending & 689 \\
Repetition & 688 \\
Ascending & 683 \\
Dashes & 672 \\
\hline
\end{tabular}

Note-Latencies are given in milliseconds.

Materials and procedure. Subjects were seated in a darkened room in front of a rear-projection screen on which all the stimuli in the experiment were presented. Exposure duration, intertrial interval, and stimulus sequencing were controlled by a Gerbrands two-channel projection tachistoscope.

Stimuli were of two types: (a) a group of four digits printed in color or (b) a rectangular patch of color covering the same area as a digit group. Both types of stimuli appeared centered on a black field. Stimuli appeared in red, blue, or green and subtended a visual angle of $1.5 \mathrm{deg}$. The following digit strings were used: $1234,3456,5678,9876,7654,5432,2468,1357$, $3579,2222,4444,7777,1776,1973,1492,5108,0236,4816$.

Trials in the experiment consisted of presentation of one of the two types of stimuli for $4 \mathrm{sec}$ during which subjects named aloud the color in which the stimulus appeared as quickly as possible. Intertrial interval was $3 \mathrm{sec}$. The subject's naming response triggered a voice-operated relay which stopped a millisecond clock started at stimulus onset. Color-naming accuracy and latency were recorded.

Prior to the beginning of the experimental trials, subjects were shown two examples of each of the three colors to be used in the experiment. In these examples, colors appeared as a row of five lower case xs printed in color and centered on a black background. Following this procedure, subjects were presented with a continuous series of 40 experimental trials. The first four trials were practice trials and consisted of two digit series trials and two patch trials. Digit stimuli used in these practice trials were not used in the subsequent experimental trials. Following the practice trials, three 12-trial blocks were given. Each block consisted of six digit and six patch trials. Each color appeared four times in each block. Stimulus order was randomized with the restrictions that no color appear on consecutive trials and no more than two digit or patch trials appear in succession. Across the three blocks, each of the digit stimuli was presented once and 18 patch stimuli were shown. Block order was balanced across subjects.

Three versions of the experimental trials were prepared. Across the three versions, each digit series appeared once in each color. One third of the subjects received each version.

\section{Results}

Color-naming errors were infrequent, occurring on only $1.2 \%$ of the digit series trials and $.8 \%$ of the patch trials. Errors consisted of production of the wrong color name or prematurely tripping the voice-operated relay with a hesitation sound such as "uh" or "er." Trials with color-naming errors were excluded from further analysis.

Mean color-naming latencies for the digit and patch trials were $613 \mathrm{msec}$ and $570 \mathrm{msec}$, respectively. Twenty three of the 27 subjects showed the effect. A repeated measures analysis of variance was performed using mean color-naming latencies with subjects and stimulus type (digits, patches) as factors. The effect of stimulus type proved significant, $F(1,26)=30.92, p<.001$.

\section{Discussion}

Experiment I demonstrates that groups of digits delay color naming when substituted for uniform patches of color in the Stroop task. Because of the design of the experiment, however, the question of the relative amount of interference produced by such "meaningful" digit groups as dates on the one hand, and less well-organized series of digits on the other, remains open. Experiment II was designed to test the degree to which several different types of four-digit groups interfere with color naming.

\section{EXPERIMENT II}

\section{Method}

Subjects. Twenty-seven undergraduates at Columbia University served individually for a single $1 / 2 \mathrm{~h}$ session and received supplementary course credit for their participation.

Materials and procedure. Except as noted below, the procedure followed that set out for Experiment I. Stimuli for the experiment were of six types: (a) dates: 1974, 1776, 1492; (b) odd or even series: 2468, 1357, 3579; (c) continuous ascending segments of the standard number series: 1234,3456 , 5678; (d) descending segments: $9876,7654,5432$; (e) repeated digits: 2222, 5555, 8888; (f) double rows of three dashes. All stimuli appeared in red, blue, or green, centered on a white field, and subtended a visual angle of approximately $4 \mathrm{deg}$.

There were $7 \mathrm{sec}$ between trial onsets. Stimuli remained visible until the subject named the color aloud, and for the remainder of the trial, the screen was blank. After six practice trials, the subject was given three 18-trial blocks. Three examples of each stimulus type appeared in each block, one in each color. Across the three blocks each digit stimulus appeared once in each color and three dash trials in each color were given. Order of presentation of the blocks was balanced across subjects. Within blocks, trials were ordered randomly with the restrictions that one trial of each type appear in each consecutive third of the block and that no color or stimulus type appear on consecutive trials.

\section{Results}

Trials on which color-naming errors or naming latencies over 2,000 msec occurred were discarded from further analysis. Such errors occurred on $.5 \%$ of the trials. No particular distribution of errors across stimulus types was noted. Mean color-naming latencies in the six conditions are shown in Table 1.

A repeated measures analysis of variance was done on mean color-naming latencies with subjects and stimulus type (dates, odd-even, ascending, descending, repetitions, and dashes) as factors. The effect of stimulus type proved significant, $F(5,130)=2.43, p<.05$. Dunnett's test was applied comparing the five digit-group types with the dash-trial mean. Only the dates and odd-even digit groups proved significantly different, both $\mathrm{p}<.01$.

\section{Discussion}

The results of Experiments I and II appear to support the contention that certain digit sequences do interfere with color naming. Groups of digits which might a priori be expected to be cohesive units, such as dates, produce the most convincing evidence of increased interference. The particular dates producing interference should, of course, vary as a function of the background of the individual tested. Those chosen for use here, 1776, 1492, and 1974 (the year the experiment was conducted) were selected for their significance to American 
college students. Other dates, such as year of birth, could be expected to produce similar effects to the degree that they are represented as cohesive units in memory.

The other group of sequences that showed significant color-naming delays, the odd-even series, provides additional evidence that grouping according to some principle other than linear order can produce a cohesive unit in memory. Here, the common juxtaposition of the set of odd digits, or even digits, appears to have formed the basis for the grouping.

The effectiveness of this second type of grouping raises the question of why the other series used in Experiment II did not produce equally long delays. The means for these groups, while greater than for the dash condition, were not significantly greater. One possible explanation is that while dates and odd-even groupings form four-, e.g., 2468, or at most five-digit series, e.g., 13579, whenever encountered, the other groupings are actually derived from the longer number sequence and do not function as separate four-digit groups. That is, 3456 is not a usual four-digit group but a segment of the longer series $12345678 \ldots$. . This situation is perhaps analogous to lifting four letters out of a longer word and comparing their cohesiveness with that of a common four-letter word. A similar lack of unity might be attributed to the repetition series. Here, a sequence of four repetitions is no more a unit than three or six repetitions and produces none of the effects to be expected from a cohesive series.

\section{REFERENCES}

Buschke, H., \& Hinrichs, J. V. Relative vulnerability of item-information in short-term storage for the missing scan. Joumal of Verbal Learning and Verbal Behavior, 1968, 7, 1043-1048.

Klein, G. S. Semantic power measured through the interference of words with color-naming. American Joumal of Psychology. 1964, 77, 576-588.

Marcel, T., \& Forrin, B. Naming latency and the repetition of stimulus categories. Journal of Experimental Psychology, $1974,103,450-460$.

Posner, M. I., \& Warren, R. E. Traces, concepts and conscious constructions. In A. W. Melton and E. Martin (Eds.), Coding processes in human memory. Washington, D.C: Winston, 1972.

Stroop, J. R. Factors affecting the speed in serial verbal reactions. Psychological Monographs, 1938, 50 (Whole No. 225), 38-48.

(Received for publication April 21, 1975.) 